\title{
Serotypes and virulence profiles of Shiga toxin-producing Escherichia coli strains isolated during 2017 from human infections in Switzerland
}

\author{
Nüesch-Inderbinen, Magdalena ; Morach, Marina ; Cernela, Nicole ; Althaus, Denise ; Jost, Marianne ; \\ Mäusezahl, Mirjam ; Bloomberg, Guido ; Stephan, Roger
}

\begin{abstract}
Since 2015, the Swiss Federal Office of Public Health registered an increase of notifications of STEC, probably due to the adoption of culture independent stx screening tests in diagnostic laboratories. This study aimed to identify the serotypes and virulence genes of 120 STEC isolated from human clinical stx positive specimens during 2017 in order to estimate any changes in serotype distribution and toxin profiles of STEC compared to the time span 2010-2014. Culturing of STEC from stool samples was achieved using the streak plate technique on MacConkey agar. We performed $\mathrm{O}$ and $\mathrm{H}$ serotyping by PCR and by micro array. Virulence genes were identified and subtyped using molecular methods, including stx1 and stx2 subtypes, and the intimin encoding gene, eae. STEC were recovered from $27.5 \%$ of the stx positive samples. STEC O157:H7 accounted for 7.5\% of all isolates, and STEC O80:H2, O91:H10/H14/H21, O103:H2/H11, and O26:H11 accounted for 36.9\% of the non-O157 strains. Forty-five isolates with stx1 variants, 47 with stx 2 variants and 28 isolates with both stx1 and stx2 variants were identified. Forty (33.3\% of all isolates) carried the subtypes associated with high pathogenic potential, stx2a, stx2c, or stx2d. The eae gene for intimin was detected in 54 strains ( $45 \%$ of all strains). Compared to 2010-2014, our data show that the proportion of the so called "top five" serogroups, STEC O26, O111, O103, and O157 declined from $53.7 \%$ to $28.3 \%$ in 2017 . The proportion of isolates with stx2a, stx2c, or stx $2 \mathrm{~d}$ decreased from $50.5 \%$ to $33.3 \%$. We also observed an increase of STEC harbouring the low pathogenic subtypes stx2b and stx2e from $12.6 \%$ to $29.2 \%$, and of eae negative STEC from $29.5 \%$ in 2010-2014 to $55 \%$ in 2017. Simultaneously, there was a sharp increase of the patients' median age from 24 years to 46.5 years. Clinical manifestations in the patients included abdominal pain without diarrhea $(22.3 \%)$, diarrhea $(77.7 \%)$, and the haemolytic-uremic syndrome (HUS) $(7.4 \%)$. Our data show that a greater number and a wider range of STEC serotypes are detected by culture-independent testing, with implications for public health services.
\end{abstract}

DOI: https://doi.org/10.1016/j.ijmm.2018.06.011

Posted at the Zurich Open Repository and Archive, University of Zurich ZORA URL: https://doi.org/10.5167/uzh-168119

Journal Article

Accepted Version

Originally published at:

Nüesch-Inderbinen, Magdalena; Morach, Marina; Cernela, Nicole; Althaus, Denise; Jost, Marianne; Mäusezahl, Mirjam; Bloomberg, Guido; Stephan, Roger (2018). Serotypes and virulence profiles of Shiga toxin-producing Escherichia coli strains isolated during 2017 from human infections in Switzerland. International Journal of Medical Microbiology : IJMM, 308(7):933-939. 
DOI: https://doi.org/10.1016/j.ijmm.2018.06.011 
1 Serotypes and virulence profiles of Shiga toxin-producing Escherichia coli strains

2 isolated during 2017 from human infections in Switzerland

3

4 Magdalena Nüesch-Inderbinen ${ }^{1}$, Marina Morach ${ }^{1}$, Nicole Cernela ${ }^{1}$, Denise Althaus ${ }^{1}$,

5 Marianne Jost ${ }^{2}$, Mirjam Mäusezahl ${ }^{2}$, Guido Bloomberg ${ }^{1}$, Roger Stephan ${ }^{1}$

6

7

$8 \quad{ }^{1}$ Swiss National Centre for Enteropathogenic Bacteria and Listeria (NENT), Institute for

9 Food Safety and Hygiene, Vetsuisse Faculty, University of Zurich, Switzerland

10

$11 \quad{ }^{2}$ Swiss Federal Office of Public Health, Division Communicable Diseases

12

13

14

$15 *$ Corresponding author:

16 Roger Stephan, Institute for Food Safety and Hygiene, Vetsuisse Faculty, University of

17 Zurich, Winterthurerstrasse 272, CH-8057 Zurich, Switzerland.

18 Phone +41 4463586 51, Fax +41 4463589 08, e-mail stephanr@,fsafety.uzh.ch

19 


\section{Abstract}

Since 2015, the Swiss Federal Office of Public Health registered an increase of notifications

of STEC, probably due to the adoption of culture independent stx screening tests in diagnostic laboratories. This study aimed to identify the serotypes and virulence genes of 120 STEC isolated from human clinical stx positive specimens during 2017 in order to estimate any changes in serotype distribution and toxin profiles of STEC compared to the time span 2010-2014. Culturing of STEC from stool samples was achieved using the streak plate technique on MacConkey agar. We performed $\mathrm{O}$ and $\mathrm{H}$ serotyping by PCR and by micro array. Virulence genes were identified and subtyped using molecular methods, including stx 1 and stx2 subtypes, and the intimin encoding gene, eae. STEC were recovered from $27.5 \%$ of the stx positive samples. STEC O157:H7 accounted for 7.5\% of all isolates, and STEC $\mathrm{O} 80: \mathrm{H} 2, \mathrm{O} 91: \mathrm{H} 10 / \mathrm{H} 14 / \mathrm{H} 21, \mathrm{O} 103: \mathrm{H} 2 / \mathrm{H} 11$, and $\mathrm{O} 26: \mathrm{H} 11$ accounted for $36.9 \%$ of the nonO157 strains. Forty-five isolates with $s t x 1$ variants, 47 with stx 2 variants and 28 isolates with both $s t x 1$ and $s t x 2$ variants were identified. Forty (33.3\% of all isolates) carried the subtypes associated with high pathogenic potential, stx $2 a, s t x 2 c$, or stx $2 d$. The eae gene for intimin was detected in 54 strains (45\% of all strains). Compared to 2010-2014, our data show that the proportion of the so called "top five" serogroups, STEC O26, O111, O103, and O157 declined from $53.7 \%$ to $28.3 \%$ in 2017 . The proportion of isolates with $s t x 2 a$, stx $2 c$, or stx $2 d$ decreased from $50.5 \%$ to $33.3 \%$. We also observed an increase of STEC harbouring the low pathogenic subtypes stx $2 b$ and stx $2 e$ from $12.6 \%$ to $29.2 \%$, and of eae negative STEC from $29.5 \%$ in $2010-2014$ to $55 \%$ in 2017 . Simultaneously, there was a sharp increase of the patients' median age from 24 years to 46.5 years. Clinical manifestations in the patients included abdominal pain without diarrhea (22.3\%), diarrhea $(77.7 \%)$, and the haemolyticuremic syndrome (HUS) (7.4\%). Our data show that a greater number and a wider range of 
44 STEC serotypes are detected by culture-independent testing, with implications for public 45 health services.

46

47

\section{Keywords}

49 STEC, human, multiplex PCR, culture, serotypes 


\section{Introduction}

Shiga toxin (Stx)-producing Escherichia coli (STEC) are etiological agents of outbreaks and of sporadic cases of human gastrointestinal illnesses which may include non-bloody or bloody diarrhea, haemorrhagic colitis (HC), and the haemolytic uremic syndrome (HUS) (Karch et al., 2005). STEC are characterized by the production of one or more Stx, which consist of two groups designated Stx1 (consisting of the three variants Stx1a, Stx1c and Stx1d) and Stx2 (composed of seven distinct variants Stx2a, Stx2b, Stx2c, Stx2d, Stx2e, Stx2f, and Stx2g). STEC associated with severe disease tend to feature variants Stx2a, Stx2c and Stx2d, whereas STEC producing Stx $2 b$ and Stx2e are linked to mild clinical symptoms or asymptomatic fecal carriage (Stephan and Hoelzle, 2000; Friedrich et al., 2002; Fuller et al., 2011). Virulence may further be increased by the presence of intimin, the product of the eae gene, which mediates attaching and effacing lesions on gastrointestinal epithelial cells (Kaper et al., 2004). STEC belonging to the serogroups O157, O26, O103, O111, and O145 constitute the so called "top five" serogroups of human pathogenic STEC in the EU and Switzerland, and are considered, together with a few others such as O91 and O113, important serogroups in public health (EFSA, 2017).

Human infection with STEC is a notifiable disease in Switzerland. Notification of confirmed cases to the federal office for public health (FOPH) is based on the isolation of STEC from faeces, or on the detection of stx 1 and/or stx 2 in faeces or from a clinical isolate of E. coli. The number of notifications of STEC in Switzerland has been increasing since 2015, possibly due to the introduction of PCR based, increasingly sensitive stx screening tests in laboratory testing practices. However, such culture-independent testing (CIDT) generally does not yield an isolate, and positive results are not always culture confirmed. From the public health point of view, the advantages of rapid and broad range pathogen detection are therefore challenged by possible loss of strain subtyping with consequent disruption of monitoring trends in 
serotype or Stx distribution (Cronquist et al., 2012). Recognition of the trends in serotypes and toxin profiles of STEC is however of great importance in order to estimate their potential for causing disease and to anticipate epidemiological changes. The aim of this study was to gain epidemiological and serotyping information on STEC isolated during 2017. Therefore, from May to December 2017, the FOPH requested all diagnostic laboratories to forward clinical materials that tested positive for stx by CIDT to the Swiss National Reference Centre for Enteropathogenic Bacteria and Listeria (NENT) for culture and further strain characterization. The isolates were analysed with regard to their serotypes, stx subtypes and presence of the eae gene. The results were compared with earlier data from Switzerland investigated over the 5-year period 2010-2014 (Fierz et al., 2017).

\section{Material and Methods}

\subsection{Sample collection}

Human stool samples that tested positive for stx using multiplex molecular methods were submitted to the NENT from May to December 2017 from clinical diagnostic laboratories distributed nationwide.

From a total of 457 submitted specimens, 436 were included for analysis after the elimination of repeat specimens (i.e., specimens obtained from the same patient). Of the 436 patients, data of age and gender were known for 431 individuals. Thereof, 240 (55.7\%) were from female and $191(44.3 \%)$ were from male patients. The median age was 46.5 years (range 0 99 years). Forty-six $(10.7 \%)$ were isolated from patients $\leq 5$ years of age.

\subsection{Strain isolation}


Specimens were cultured on MacConkey agar using the streak plate technique. From each plate, six individual colonies, if possible of different morphology, were picked und subcultured on sheep blood agar (Difco ${ }^{\mathrm{TM}}$ Columbia Blood Agar Base EH; Becton Dickinson AG, Allschwil, Switzerland). Isolates that were confirmed to possess stx (stx 1 and/or stx2) by real-time PCR (LightCycler R 2.0 Instrument, Roche Diagnostics Corporation, Indianapolis, IN, USA) (EURL, 2013a) were selected for further analysis. From plates yielding more than one stx positive colony, one isolate was randomly chosen for subsequent characterization. Proportions of STEC in stool samples were defined as the numbers of stx positive colonies among six E. coli colonies.

\subsection{Serotyping}

Strains were examined by PCR for the presence of genes associated with 14 selected serogroups including the top-five serogroups, namely O26, O45, O55, O80, O91, O103, O104, O111, O113, O121, O128, O145, O146, and O157 (Perelle et al., 2004; EURL, 2013a; EURL, 2014; Soysal et al., 2016). Strains were tested for the presence of flagellar genes related to H2, H4, H7, H8, H10, H11, H19, H21, H25, and H28 (Mora et al., 2012; EURL, 2013b; Beutin et al., 2015; Alonso et al., 2017). Strains belonging to other O groups and H types were serotyped using the Alere ${ }^{\mathrm{TM}}$ E. coli SeroGeno typing AS-1 kit (Alere Technologies, Jena, Germany).

\subsection{Virulence markers}

The identification of $s t x 1$ subtypes (stx $1 a, s t x 1 c, s t x 1 d)$ and $s t x 2$ subtypes $(s t x 2 a, s t x 2 b, s t x 2 c$, stx2d, stx2e, stx2g) was carried out by conventional PCR amplification (Scheutz et al., 2012). Screening of the strains for eae was performed by real-time PCR according to the guidelines of the European Union Reference laboratory (EURL, 2013a). 


\section{Results}

\subsection{Recovery rate}

127 Out of a total of 436 human fecal specimens that tested positive by multiplex PCR for the

128 presence of stx, 120 samples yielded an STEC isolate for further characterisation, amounting

129 to a recovery rate of $27.5 \%$.

130 Categorising the 120 samples into those with high numbers of stx positive colonies (five or

131 six positive colonies per sample), those with intermediate numbers (three or four positive

132 colonies) and those with low numbers (one or two colonies), resulted in $46(38.3 \%)$ stool

133 samples with a high proportion of STEC colonies, $35(29.2 \%)$ with an intermediate proportion, and $39(32.5 \%)$ stool samples with a low proportion of STEC, respectively Strains exhibiting high or low colony numbers are listed in Table 1.

\subsection{Serological diversity}

138 Twenty-five different O-serogroups were identified among the 120 STEC isolates, in addition 139 to 17 O-non-typeable (Ont) serogroups, and 4 ambiguous results. Eighteen different H-types

140 were determined, including two non-typeable H-types and two ambiguous results. An

141 overview of the serotypes is given in Table 1.

142 Among the 120 isolates, 9 (7.5\%) were O157:H7, and 111 (92.5\%) were non-O157 STEC

143 strains. Together with STEC O157, the top five serogroups were represented by STEC O103

$144(\mathrm{n}=11), \mathrm{O} 145(\mathrm{n}=6)$, and $\mathrm{O} 26(\mathrm{n}=8)$, amounting to $28.3 \%$ of the isolates. No STEC O111

145 were detected. Isolates belonging to O80:H2 (n=11), and O91:H10/H14/H21 (n=11)

146 accounted each for $9.2 \%$ of the isolates, respectively. Other serotypes included

147 O174:H2/H8/H21 (n=7; 5.8\%), and O146:H21 $(\mathrm{n}=6 ; 5 \%)$ (Table 1). Other serotypes were 148 represented by four or less STEC isolates (Table 1). 


\subsection{Distribution of virulence genes among the serotypes}

151 Of the 120 STEC strains, $45(37.5 \%)$ carried stxl genes only: stxla $(\mathrm{n}=35)$, stxlc $(\mathrm{n}=9)$

152 and stxld $(\mathrm{n}=1)$. Forty-seven strains $(39.1 \%)$ carried stx2 genes only: stx $2 a(\mathrm{n}=14)$, stx $2 b(\mathrm{n}$

$153=14)$, stx2c $(\mathrm{n}=5)$, stx2d $(\mathrm{n}=12)$, and stx2e $(\mathrm{n}=2)$. Twenty-eight $(23.3 \%)$ harboured

154 combinations of $s t x 1$ and stx 2 genes. Forty (33.3\% of all isolates) carried the subtypes

155 associated with high pathogenic potential, stx2a, stx $2 c$, or st $x 2 d$ (Table 1). The

156 majority thereof $(n=25 / 62.5 \%$ of the 40 strains $)$ were associated with O80:H2 $(n=11)$,

$157 \quad \mathrm{O} 157: \mathrm{H} 7(\mathrm{n}=9)$ and O145:H28 $(\mathrm{n}=5)$.

158 Thirty-five (29.2\%) isolates harboured the low pathogenic subtypes stx $2 b$ and stx $2 e$ and were 159 mainly associated to the serogroup O146 and Ont serogroups.

160 The eae gene encoding intimin was detected in 54 strains ( $45 \%$ of all strains). Thereof, 31

$161 \quad(57.4 \%$ of the eae positive strains) were associated with $\operatorname{stx} 2 a, \operatorname{stx} 2 c$, or $\operatorname{stx} 2 d$, and one (4.3\%)

162 with $s t x 2 b$. The remaining $22(40.7 \%)$ of the eae positive isolates carried stx la alone (Table

$1631)$.

164 The majority (25 strains, $80.6 \%$ ) of the eae positive strains harbouring stx2 subtypes

165 belonged to STEC O80:H2, O145:H28, and O157:H7. By contrast, of the 66 (55\% of all

166 strains) that tested negative for eae, nine (13.6\% of all eae negative strains) harboured stx $2 a$,

$167 s t x 2 c$, or $s t x 2 d$, while $34(51.5 \%)$ were associated with $s t x 2 b$ or $s t x 2 e$. The remaining 23

$168 \quad(34.8 \%)$ carried stxla stxlc or stxld alone (Table 1).

169 The distribution of serogroups and genotypes compared to earlier data from 2010-2014 (Fierz

170 et al., 2017) is illustrated in Figure 1.

171 


\subsection{Relationship between STEC type, age of patients, clinical symptoms, and}

173

174

175

176

177

178

179

180

181

182

183

184

185

186

187

188

189

190

191

192

193

194

195

196

197

\section{proportions of $s t x$ positive $E$. coli in stool samples}

Patients were classified into four groups, according to their age at the time of sampling. Age group 1 consisted of infants and children $\leq 5$ years of age $(n=14)$, group 2 contained children and young adults between 6 and 17 years $(n=16)$. Group 3 consisted of adult patients between 18 and 60 years $(n=56)$, and group 4 of patients $>60$ years $(n=33)$. For one patient, the age was unknown (Table 1). Clinical data were provided for $94(78.3 \%)$ of the patients. Abdominal pain without diarrhea (AP) was reported for $21(22.3 \%)$ of the patients. The majority (77.7\%) suffered from diarrhea (D). HUS was present in 7 (7.4\%) of the patients, six thereof with D, and one with AP. Two further patients (2.1\%) had presumptive HUS with acute kidney failure (AKF). Nineteen (20.2\%) patients were hospitalized (two patients with AP only and 17 patients with D).

The distribution of STEC serotypes and of stx2 and eae genes among the patients' age groups is listed in Table 1. STEC belonging to the top five serogroups were found in $71.2 \%$ of the STEC infected children from age group 1 and in $56.3 \%$ from age group 2 . Infections due to the top five STEC serotypes were less frequent among age group 3 (16.1\% of the patients), and age group 4 (18.2\% of the patients). By contrast, of the two most prevalent serogroups from this study, STEC O80 was isolated more frequently from patients in age group 4 (15.2\% of the patients) than from patients in groups 1 (7.1\%), group $2(6.3 \%)$ and group $3(7.1 \%)$, respectively. STEC O91 was detected only among isolates from patients of age groups 3 and 4, accounting for $16.1 \%$ and $6 \%$ of the infections, respectively. STEC harbouring $s t x 2 a / s t x 2 d / s t x 2 c$ were frequent among patients from age groups 1,2 and $4(50 \%, 43.8 \%$, and $42.4 \%$ respectively), and least frequent among patients from age group $3(19.6 \%)$. STEC containing stx $2 b /$ stx $2 e$ was more frequent among patients from age groups 3 and $4(33.9 \%$ and $33.3 \%$, respectively), compared to patients from age groups 1 and $2(7.1 \%$ and $25 \%$, 
respectively). Similarly, eae positive STEC were observed at higher rates among patients from groups 1 and $2(85.7 \%$ and $62.5 \%$, respectively) than among those from groups 3 and 4 (30.4\% and 42.2\%, respectively). By contrast, eae negative STEC were remarkably less frequent in isolates from patients of age groups 1 and $2(14.3 \%$ and $37.5 \%$, respectively) than in those from group 3 and 4 (69.6\% and 57.6\%, respectively). The distribution of STEC serogroups and of stx2 and eae genes among the patients' age groups is illustrated in Figure $2 \mathrm{~A}$ and $\mathrm{B}$, respectively.

STEC serotypes and virulence genes associated with patients with abdominal pain only, diarrhea, HUS, and with patients that were hospitalised are listed in Table 1. STEC belonging to the top five serogroups were found in $14.3 \%$ of 21 patients with AP, in $30.1 \%$ of 73 patients with D and in $42.9 \%$ of 7 patients with HUS (Table 1). The top five serogroups were furthermore associated with $31.6 \%$ of 19 hospitalised patients. STEC O80 was isolated from $4.8 \%$ of patients with AP and $12.3 \%$ of patients with D. Moreover, STEC O80:H2 was associated with one case of HUS, two cases of AKF and isolated from $21 \%$ of hospitalised patients (Table 1). STEC O91 was detected at similar rates among patients with AP and D ( $14.3 \%$ and $9.6 \%$, respectively), in one HUS case and in $15.8 \%$ of hospitalised patients. STEC harbouring stx $2 a / s t x 2 d / s t x 2 c$ were less frequent among patients with AP (14.3\%), than among isolates from patients with D (41\%) and HUS patients (100\%), and were found in $68.4 \%$ of hospitalised patients. Similarly, eae positive STEC were observed at a lower rate in patients with AP (23.8\%) than among patients with D (47.9\%) or HUS (85.7\%), and hospitalised patients (63.2\%) (Table 1). By contrast, STEC carrying stx2b/stx2e were more frequent among patients with AP (57.2\%) than among patients with D (21.9\%), and absent among HUS patients. 
221 Finally, eae negative STEC were accountable for $76.2 \%$ of patients with AP only, and for

$22252.1 \%$ and $14.3 \%$ of patients with D and HUS, respectively. Furthermore, eae negative STEC

223 were recovered from $36.8 \%$ of hospitalised patients.

224 The distribution of STEC serogroups and of stx2 variants and eae genes among patients with

225 AP, D, HUS, and hospitalized patients is illustrated in Figure 2C and D, respectively.

226 The proportions of STEC among E. coli isolated from the patients' stool samples varied

227 according to serotypes and virulence genes. The majority of the STEC O145, STEC O157,

228 and STEC O91 isolates $(66.7 \%, 55.6 \%$, and 54.5\%, respectively) were found in high numbers

229 in the stool samples, whereas high proportions were less frequent for serogroups O26 (50\%)

230 and $\mathrm{O} 103$ (36.4\%), and remarkably less (28.1\%), for isolates belonging to serogroups other

231 than the top five, O80 or O91 (Figure 2E). STEC isolates harbouring stx2a/stx2c/stx2d were

232 observed more frequently in higher proportions than those harbouring stx $2 b / s t x 2 e$ ( $50 \%$ and

$23334.3 \%$, respectively). Similarly, eae positive STEC were found more frequently in high

234 proportions among E. coli from patients' stool samples than eae negative STEC (44.4\% and

$23533.3 \%$, respectively, Figure 2F).

236 Among patients with AP, the proportion of STEC in stool was high for $33.3 \%$ and low for

$23747.6 \%$. Among patients with D and HUS, proportions were high for $37 \%$ and $42.9 \%$,

238 respectively, and low for $32.9 \%$ and $28.6 \%$ (Table 1 ).

\section{Discussion}

242 Since 2015, the Swiss FOPH has registered an increase of notifications of STEC related

243 infections compared to previous years, with the increasing use of stx screening tests driving

244 this trend (Hächler and Stephan, 2015). In order to estimate any changes in serotype 
distribution and toxin profiles of STEC, this study aimed to identify the serotypes and virulence genes of 120 STEC isolated from human clinical specimens during 2017.

247 The five most common serogroups were O157, O103, O26, O91 and O80, with E. coli

248 O157:H7 accounting for 7.5\% of the STEC strains. By comparison, during 2000-2009,

$24930.6 \%$ of the STEC strains isolated from humans in Switzerland were E. coli $\mathrm{O} 157: \mathrm{H} 7$, and 250 during 2010-2014 this rate further decreased to 19\% (Käppeli et al., 2011; Fierz et al., 2017).

251 Thus, as reported for other countries in the EU, the proportion of STEC O157 isolated from

252 humans continues to decrease in Switzerland (EFSA, 2017). Similarly, the proportion STEC

253 O145 which belongs to the top five serogroups in Europe (EFSA, 2017), decreased from

$25412.6 \%$ during 2010-2014 to 5.4\% in 2017 (Fierz et al., 2017). By contrast, with regard to the serogroups that do not belong to the top five, we observed an increase of STEC O80 from $6.3 \%$ to $9.2 \%$ and of STEC O91 from 3.2\% to 9.2\%, compared to 2010-2014 (Fierz et al., 2017). In particular, the increase of STEC O80:H2 is noteworthy. This hypervirulent, multidrug resistant serotype harbours genetic characteristics of a hybrid STEC/ extraintestinal

259 pathogenic E. coli (ExPEC) pathotype and has recently emerged in France and Switzerland associated with severe disease including bacteremia and HUS (Soysal et al., 2016; Fierz et al., 2017; Nüesch-Inderbinen et al., 2018).

262 Compared to the study period 2010-2014, we also observed a remarkable decrease in the 263 percentage of STEC harbouring stx2 variants that are associated with severe disease (i.e., 264 stx $2 a$, stx $2 c$, stx $2 d$ ) from $50.5 \%$ to $33.3 \%$, and of eae positive STEC from $70.5 \%$ to $45 \%$.

265 Simultaneously, we noted a sharp increase from $12.6 \%$ to $29.2 \%$, of STEC associated with $s t x 2 b$ and stx $2 e$, variants that are linked to mild clinical symptoms and asymptomatic carriage

267 (Stephan and Hoelzle, 2000; Fuller et al., 2011), and to eae negative STEC from 29.5\% to $55 \%$. Correlating to this development, the patients' age median increased from 24 years to 
46.5 years, and the proportion of patients $\leq 5$ years dropped from $43.2 \%$ in $2010-2014$ to $10.7 \%$ in 2017.

271 The present study analysed STEC cultured stool samples that were positive for stx by PCR 272 screening. The relatively low success rate of $27 \%$ for isolation of STEC by culture compared 273 with other studies 72\%-96.5\% (Friedrich et al., 2002; Tunsjø et al., 2015) may be explained 274 by the loss of stx genes over time due to transport conditions or time delay to culture in the 275 reference laboratory. A further explanation is that the concentration of STEC in some of the stool samples may have been too low to allow detection of isolates. Alternatively, the low

277 recovery may have been caused by the use of PCR to directly target stx genes in faeces. Free 278 stx-carrying bacteriophages have been detected at a prevalence of $62 \%$ in fecal samples of 279 healthy people and may thus be responsible for some of the stx positive stool samples 280 (Martinez-Castillo et al., 2013; Urdahl et al., 2013). Therefore, caution should be employed 281 when diagnosing disease on the basis of stx positive results obtained directly from faecal 282 samples.

283 A definite attribution of the STEC isolates from this study to disease was precluded by the 284 lack of sufficient information on co-detection of pathogens other than STEC by multiplex PCR based CIDT. Overall, it cannot be excluded that a certain number of the isolated STEC were not the actual etiological agent of disease. Indeed, ten $(8.3 \%)$ of the STEC strains identified in this study belong to serotypes detected previously in faecal samples of healthy humans including O8:H25, O128:H10, O146:H21, O146:H28, and O174:H8 (Stephan and

289 Untermann, 1999; Stephan and Schumacher, 2001; Urdahl et al., 2013).

290 Our data are supportive of previous studies that established an association of eae negative 291 STEC with severe clinical symptoms including HUS (Beutin et al., 2004). However, further studies are needed in order to correlate the presence of infrequently detected STEC serotypes 
and $s t x 2 b / s t x 2 e$ harbouring STEC with the severity of disease and, ultimately, to distinguish

294 public health relevant infections from the non-relevant.

295 Conclusively, in spite of the increased notification of STEC during 2017, there was no

296 evidence for an outbreak situation. Our data are suggestive of an ongoing trend towards a

297 wider spectrum of serologically different STEC types most likely due to the introduction of

298 PCR based, increasingly sensitive stx screening tests in laboratory testing practices. This

299 study emphasises the importance of combining molecular methods of detection with classical

300 culture techniques to enable the detection of emerging STEC serotypes or outbreak situations.

301 Moreover, recognition of the trends in serotypes and toxin profiles of STEC is of great

302 importance in order to estimate epidemiological changes.

303

304 Funding

305 This work was partly supported by the Swiss Federal Office of Public Health, Division

306 Communicable Diseases.

307

\section{Conflicts of interest}

309 None to declare.

Acknowledgements

313 The authors thank all contributing diagnostic laboratories. 


\section{References}

315

316

Alonso, C. A., Mora, A., Díaz, D., Blanco, M., González-Barrio, D., Ruiz-Fons, F., Simón, C., Blanco, J., Torres, C., 2017. Occurrence and characterization of stx and/or eaepositive Escherichia coli isolated from wildlife, including a typical EPEC strain from a wild boar. Vet. Microbiol. 207, 69-73. doi=10.1016/j.vetmic.2017.05.028.

Beutin, L., Krause, G., Zimmermann, S., Kaulfuss, S., Gleier, K., 2004. Characterization of Shiga toxin-producing Escherichia coli strains isolated from human patients in Germany over a 3-year period. J. Clin. Microbiol. 42, 1099-1108.

Beutin, L., Delannoy, S., Fach, P., 2015. Genetic Diversity of the $f l i C$ genes encoding the flagellar antigen H19 of Escherichia coli and application to the specific identification of enterohemorrhagic E. coli O121:H19. Appl. Environ. Microbiol. 81, 4224-4230. DOI=10.1128/AEM.00591-15.

Cronquist, A. B., Mody, R. K., Atkinson, R., Besser, J., Tobin D’Angelo, M., Hurd, S., Robinson, T., Nicholson, C., Mahon, B. E., 2012. Impacts of culture-independent diagnostic practices on public health surveillance for bacterial enteric pathogens. Clin. Infect. Dis. 54 Suppl 5, S432-439. DOI=10.1093/cid/cis267.

EFSA (European Food Safety Authority) and ECDC (European Centre for Disease Prevention and Control), 2017. The European Union summary report on trends and sources of zoonoses, zoonotic agents and food-borne outbreaks in 2016. EFSA J. 15(12):5077, 228 pp. https://doi.org/10.2903/j.efsa.2017.5077.

European Union Reference Laboratory (EURL), 2013a. Identification and characterization of verocytotoxin-producing Escherichia coli (VTEC) by real time PCR amplification of the main virulence genes and the genes associated with the serogroups mainly associated with severe human infections. EU-RL 
VTEC_Method_02_Rev 0. Available online at:

European Union Reference Laboratory (EURL), 2013b. Detection and identification of verotoxin-producing Escherichia coli (VTEC) O104:H4 in food by real time PCR. EU-RL VTEC_Method_04_Rev 1. Available online at: http://old.iss.it/binary/vtec/cont/EU_RL_VTEC_Method_04_Rev_1.pdf.

European Union Reference Laboratory (EURL), 2014. Identification of the VTEC serogroups mainly associated with human infections by conventional PCR amplification of O-associated genes. EU-RL VTEC_Method_03_Rev 01.

Fierz, L., Cernela, N., Hauser, E., Nüesch-Inderbinen, M., Stephan, R. 2017. Characteristics 
363 Käppeli, U., Hächler, H., Giezendanner, N., Cheasty, T., Stephan, R., 2011. Shiga toxin364 producing Escherichia coli O157 associated with human infections in Switzerland, 20002009. Epidemiol. Infect. 139, 1097-1104. DOI=10.1017/S0950268810002190.

Karch, H., Tarr, P. I., Bielaszewska, M., 2005. Enterohaemorrhagic Escherichia coli in human medicine. Int. J. Med. Microbiol. 295, 405-418. DOI=10.1016/j.ijmm.2005.06.009.

Martinez-Castillo, A., Quirós, P., Navarro, F., Miró, E., Muniesa, M. 2013. Shiga toxin 2encoding bacteriophages in human fecal samples from healthy individuals. Appl. Environ. Microbiol. 79, 4862-4868. DOI=10.1128/AEM.01158-13.

Mora, A., López, C., Dhabi, G., López-Beceiro, A. M., Fidalgo, L. E., Díaz, E. A., MartínezCarrasco, C., Mamani, R., Herrera, A., Blanco, J. E., Blanco, M., Blanco, J., 2012. 185-192. DOI=10.1016/j.mcp.2003.12.004. 
Soysal, N., Mariani-Kurkdjian, P., Smail, Y., Liguori, S., Gouali, M., Loukiadis, E., Fach, P., Bruyand, M., Blanco, J., Bidet, P., Bonacorsi, S., 2016. Enterohemorrhagic Escherichia coli hybrid pathotype O80:H2 as a new therapeutic challenge. Emerg. Infect. Dis. 22, 1604-1612. doi=10.3201/eid2209.160304

Stephan, R., Hoelzle, L. E., 2000. Characterization of Shiga toxin type 2 variant B-subunit in Escherichia coli strains from asymptomatic human carriers by PCR-RFLP. Lett. Appl. Microbiol. 31, 139-142.

Stephan, R., Schumacher, S. 2001. Resistance patterns of non-O157 Shiga toxin-producing Escherichia coli (STEC) strains isolated from animals, food and asymptomatic human

Stephan, R., Untermann, F., 1999. Virulence factors and phenotypical traits of verotoxin403 producing Escherichia coli strains isolated from asymptomatic human carriers. J. Clin. carriers in Switzerland. Lett. Appl. Microbiol. 32, 114-117. DOI=10.1046/j.1472765x.2001.00867.x. Microbiol. 37, 1570-1572.

Tunsjø, H. S., Kvissel, A. K., Follin-Arbelet, B., Brotnov, B. M., Ranheim, T. E., Leegaard, T. M. 2015. Suitability of $s t x$-PCR directly from fecal samples in clinical diagnostics of STEC. APMIS. 123, 872-878. DOI=10.1111/apm.12428. 


\section{Figure legends}

415 Figure 1: Comparative distribution of serogroups and genotypes of STEC isolated during $416 \quad 2010-2014$ and in 2017 from humans.

417 A: Percentage of the "top five" STEC serogroups and other selected STEC serogroups 418 isolated during 2010-2014 and in 2017.

419 B: Percentage of STEC harbouring stx 1 and stx2 subtypes, and percentage of eae positive and 420 eae negative STEC isolated during 2010-2014 and in 2017.

421 Figure 2: Relationship between STEC type, patients' age, clinical symptoms, and proportions

422 of STEC types among E. coli in stool samples.

423 A: Percentage of patients by age group infected with isolates belonging to the top five STEC serogroups (O26, O103, O111, O145, or O157), STEC O80, STEC O91, or other STEC 425 serogroups.

426 B: Percentage of patients by age group infected with STEC harbouring stx 2 virulence genes 427 associated with high pathogenicity $(s t \times 2 a / s t x 2 c / s t x 2 d)$, stx 2 genes associated with milder 428 symptoms $($ stx2b/stx2e), eae positive $(e a e+)$ STEC, and eae negative (eae -) STEC.

429 C: Distribution of STEC serogroups isolated among patients with abdominal pain only (AP), 430 diarrhea (D) or the hemorrhagic uremic syndrome (HUS), and from hospitalised patients (H).

431 D: Distribution of STEC harbouring stx2 virulence genes, and percent eae + and eae - STEC 432 among patients with AP, D or HUS and among hospitalised patients.

433 E: Percentage of selected STEC serogroups present in high or low proportions among E. coli 434 isolated from human stool samples. Stool samples with five or six stx positive colonies out of 435 six E. coli colonies were classified as high. Samples with one or two STEC per six E. coli 436 colonies were defined as low proportion. 
437 F: Percentage of STEC harbouring stx2 subtypes, and percentage of eae positive and eae

438 negative STEC present in high or low proportions among E. coli isolated from human stool 439 samples.

440

441

442

443 